\title{
Mesohepatectomía, una alternativa para el manejo del hepatocarcinoma en paciente no cirrótico: serie de casos
}

\author{
Mesohepatectomy, an alternative for the management of hepatocellular \\ carcinoma in non-cirrhotic patient: case series
}

\author{
Luis Manuel Barrera-Lozano $\mathbb{D}^{\mathbb{D}}$, Jaime Alberto Ramírez ${ }^{1} \mathbb{D}$, Jorge Andrés Becerra ${ }^{1} \mathbb{D}$, \\ Cristian Leonardo Muñoz ${ }^{2} \mathbb{D}$, Diana Catalina Pineda ${ }^{\mathbb{D}}$, Jorge Iván Gutiérrez-Montoya ${ }^{4} \mathbb{D}$
}

1 Médico, especialista en Cirugía general y Cirugía de trasplantes, Unidad Funcional de Trasplantes y Enfermedades Digestivas, Hospital San Vicente Fundación, Rionegro, Colombia.

2 Médico, especialista en Cirugía general, Unidad Funcional de Trasplantes y Enfermedades Digestivas, Hospital San Vicente Fundación, Rionegro, Colombia.

3 Médico, especialista en Cirugía general, Unidad Funcional de Trasplantes y Enfermedades Digestivas, Hospital Universitario San Vicente Fundación, Medellín, Colombia.

4 Médico, especialista en Cirugía general y Cirugía de trasplantes, Unidad Funcional de Trasplantes y Enfermedades Digestivas, Hospital Universitario San Vicente Fundación, Medellín, Colombia.

\section{Resumen}

Introducción. La resección quirúrgica es el tratamiento de elección de las neoplasias primarias y secundarias del hígado. Los pacientes con hepatocarcinoma de los segmentos centrales representan un reto, siendo la hepatectomía extendida la técnica más usada, sin embargo, el riesgo postquirúrgico de falla hepática es alto, dado que la resección puede comprometer entre el 65 \% y el 80 \% del volumen hepático. La mesohepatectomía es una alternativa que permite dejar un volumen hepático residual suficiente. El objetivo de este trabajo es presentar nuestra experiencia en el tratamiento de pacientes con hepatocarcinomas en segmentos centrales a quienes se les realizó mesohepatectomía.

Serie de casos. Se presentan tres pacientes no cirróticos, con hepatocarcinoma en los segmentos 4, 5 y 8, que fueron atendidos en el Hospital San Vicente Fundación, en las sedes de Medellín y de Rionegro, entre 2018 y 2020.

Resultados. La mesohepatectomía se realizó mediante ligadura selectiva de los pedículos del segmento 4 y del sector anterior derecho. Se utilizó aspirador ultrasónico y endograpadora para la transección hepática. La duración de la maniobra de Pringle varió entre 16 y 43 minutos. El sangrado promedio fue de $1000 \mathrm{ml}$. Solo un paciente presentó fuga biliar tipo B. No hubo mortalidad a 30 días.

Conclusiones. La mesohepatectomía es una alternativa segura para pacientes con tumores en los segmentos centrales, que permite disminuir el riesgo de falla hepática luego de la resección.

Palabras clave: hepatocarcinoma; hepatectomía; central; extendida; falla hepática; postoperatoria; cirrosis hepática.

Fecha de recibido: 16/06/2021 - Fecha de aceptación: 28/08/2021 - Publicación en línea: 09/11/2021

Correspondencia: Luis Manuel Barrera-Lozano, Hospital San Vicente Fundación Rionegro, Vereda La Concepción, Km 2,3 Vía Llanogrande - Aeropuerto. Rionegro, Colombia. Teléfono: (57-4) 4482626 extensión 3423. Correo: luis.barrera@sanvicentefundacion.com

Citar como: Barrera-Lozano LM, Ramírez JA, Becerra JA, Muñoz CL, Pineda DC, Gutiérrez-Montoya JI. Mesohepatectomía, una alternativa para el manejo del hepatocarcinoma en paciente no cirrótico: serie de casos. Rev Colomb Cir. 2022;37:96-105.

https://doi.org/10.30944/20117582.928

Este es un artículo de acceso abierto bajo una Licencia Creative Commons - BY-NC-ND https://creativecommons.org/licenses/by-nc-nd/4.0/deed.es 


\begin{abstract}
Introduction. Surgical resection is the treatment of choice for primary and secondary neoplasms of the liver. Patients with central segment hepatocarcinoma represent a challenge, with extended hepatectomy being the most widely used technique. However, the postsurgical risk of liver failure is high since resection can compromise between $65 \%$ and $80 \%$ of liver volume. Mesohepatectomy is an alternative that allows a sufficient residual liver volume to be left. The objective of this work is to present treatment of patients with central segment hepatocarcinoma.
\end{abstract}

Clinical cases. Three non-cirrhotic patients are presented, with hepatocarcinoma in segments 4, 5 and 8, who were treated at the San Vicente Fundación Hospital in Medellín and Rionegro, between 2018 and 2020.

Results. Mesohepatectomy was performed by selective ligation of the pedicles of segment 4 and the right anterior sector. An ultrasonic aspirator and endostapler were used for liver transection. The duration of the Pringle maneuver ranged from 16 to 43 minutes. The average bleeding was $1000 \mathrm{cc}$. Only one patient had type B bile leakage. There was no 30-day mortality.

Conclusions. Mesohepatectomy is a safe alternative for patients with tumors in the central segments, which reduces the risk of liver failure after resection.

Keywords: hepatocarcinoma; hepatectomy; central; extended; liver failure; postoperative; hepatic cirrhosis.

\section{Introducción}

McBride y Wallace describieron por primera vez en 1976 una resección exitosa de un tumor de $7 \mathrm{~cm}$ en los segmentos centrales del hígado en un niño de 12 años ${ }^{1}$. Dicha técnica también conocida como mesohepatectomía $(\mathrm{MH})^{2}$, se caracteriza por la ligadura de los ramos portales izquierdos hacia el segmento 4 , el ramo portal del sector anterior derecho y la vena suprahepática media, resecando los segmentos 4,5 y 8 en su totalidad ${ }^{3,4}$. Esta técnica permite preservar entre el 35 y el $50 \%$ del volumen hepático residual funcional ${ }^{5}$, porcentaje mucho mayor si se compara con la hepatectomía extendida (HE), en la que solo queda entre el 20 y el $30 \%$ del volumen hepático, aumentando el riesgo de falla hepática posterior a la hepatectomía.

El hecho de dejar mayor remanente hepático durante la mesohepatectomía, favorece a largo plazo la resección de recurrencias en el hígado residual, que es el tratamiento de elección de los hepatocarcinomas en pacientes no cirróticos.

A pesar de dichos beneficios, esta técnica es más demandante que una hepatectomía extendida, tiene mayor riesgo de sangrado intraoperatorio $y$ de fugas biliares del lecho de resección, por lo que muchos centros la han dejado como una alter- nativa casi anecdótica para el tratamiento de los tumores de los segmentos centrales.

Actualmente, en Latinoamérica solo se encuentran reportes de casos aislados de mesohepatectomías, tanto en enfermedad benigna (cistadenomas biliares) como maligna primaria y metastásica ${ }^{6-8}$. A la fecha en Colombia no encontramos publicaciones de series de casos con esta técnica para el manejo de una misma patología, por lo que el objetivo de este trabajo es presentar una serie de tres casos de pacientes con hepatocarcinoma en los segmentos centrales, sobre hígado no cirrótico, quienes por edad y tamaño de las lesiones no fueron candidatos a trasplante hepático y cuyo volumen del hígado remanente probablemente era insuficiente.

\section{Caso 1}

Masculino de 83 años con antecedente de hipertensión arterial (HTA), enfermedad pulmonar obstructiva crónica (EPOC), colitis ulcerativa y enfermedad coronaria, que consultó por dolor abdominal irradiado al dorso y pérdida de peso. En la ecografía se encontró una masa de $9 \mathrm{~cm}$ en el lóbulo hepático derecho por lo que se realizó una Resonancia Nuclear Magnética (RNM) de abdomen contrastada que mostró un hígado 
no cirrótico con un hepatocarcinoma de $9 \mathrm{~cm}$ en los segmentos 4a, 5 y 8 (figura 1). Otros estudios practicados al paciente mostraron: bilirrubina total (BT) de 0,7 mg/dL, albúmina de 3,7 g/ $\mathrm{dL}$, fosfatasa alcalina (FA) de $78 \mathrm{U} / \mathrm{L}$, aspartato amino transferasa (AST) de $33 \mathrm{U} / \mathrm{L}$, alanino aminotransferasa (ALT) de 59 U/L, INR de 1,01, anticuerpo para virus de hepatitis C (AcVHC) negativo, antígeno de superficie para virus de hepatitis B (AgsHBV) negativo, hemoglobina 12,9 $\mathrm{g} / \mathrm{dL}$, plaquetas $212,000 \mathrm{mcL}$, alfa fetoproteina (AFP) $2,8 \mathrm{ng} / \mathrm{mL}$, antígeno carcinoembrionario (ACE) 3,3 ng/mL, antígeno carbohidrato 19-9 (Ca 19-9) de 33,9 U/mL, tomografía computarizada (TC) de tórax y gammagrafía ósea negativas para metástasis. Dado el tamaño de la lesión, la edad del paciente y el volumen hepático residual funcional insuficiente en caso de una hepatectomía derecha extendida, se decidió proceder con una mesohepatectomía.
Se practicó una incisión de Mackuuchi, liberando la totalidad de los ligamentos hepáticos, se realizó colecistectomía retrógrada, y disección de la arteria hepática propia, y sus ramas derecha e izquierda. Con la movilización del conducto hepático común hacia medial usando el cístico como pivote, se logró disecar y exponer los ramos portales y arteriales de los sectores anterior y posterior derecho. Se ligó la arteria y vena porta sectorial anterior derecha y se disecó y reparó la vena suprahepática media, izquierda y derecha por separado. Se realizó la resección hepática con pinza Harmonic ${ }^{\circledR}$ a través de las líneas de demarcación isquémica dada para el sector anterior derecho, y se grapó finalmente la vena suprahepática media a casi $2 \mathrm{~cm}$ de su llegada a la vena cava inferior (VCI) (figura 2). El tiempo operatorio fue de 180 minutos y el de la maniobra de Pringle de 16 minutos. El sangrado total transoperatorio fue de $800 \mathrm{ml}$, sin requerir transfusiones. El margen macroscópico de los bordes fue de $1 \mathrm{~cm}$ (figura 3).

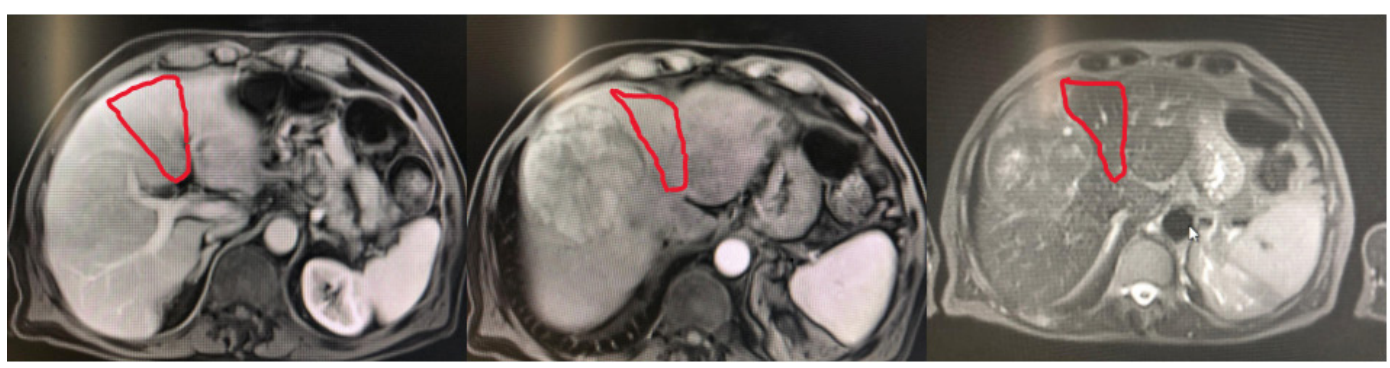

Figura 1. Resonancia nuclear magnética de abdomen. Se observa un hepatocarcinoma de $9 \mathrm{~cm}$ en el sector anterior derecho con compromiso parcial del segmento 4 (señalado en rojo).

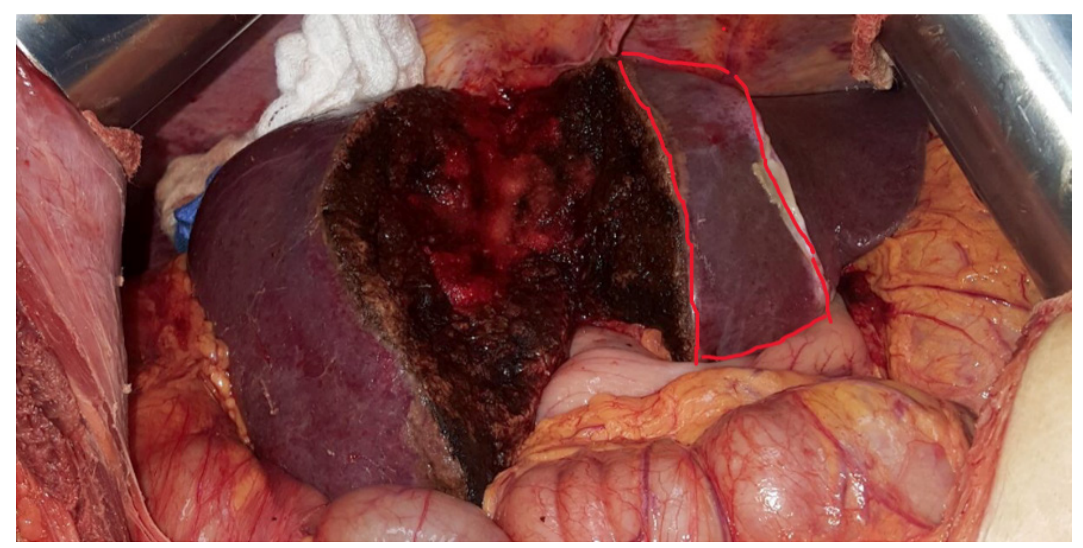

Figura 2. Resección hepática de sector anterior derecho con preservación parcial segmento 4 (señalado en rojo). 

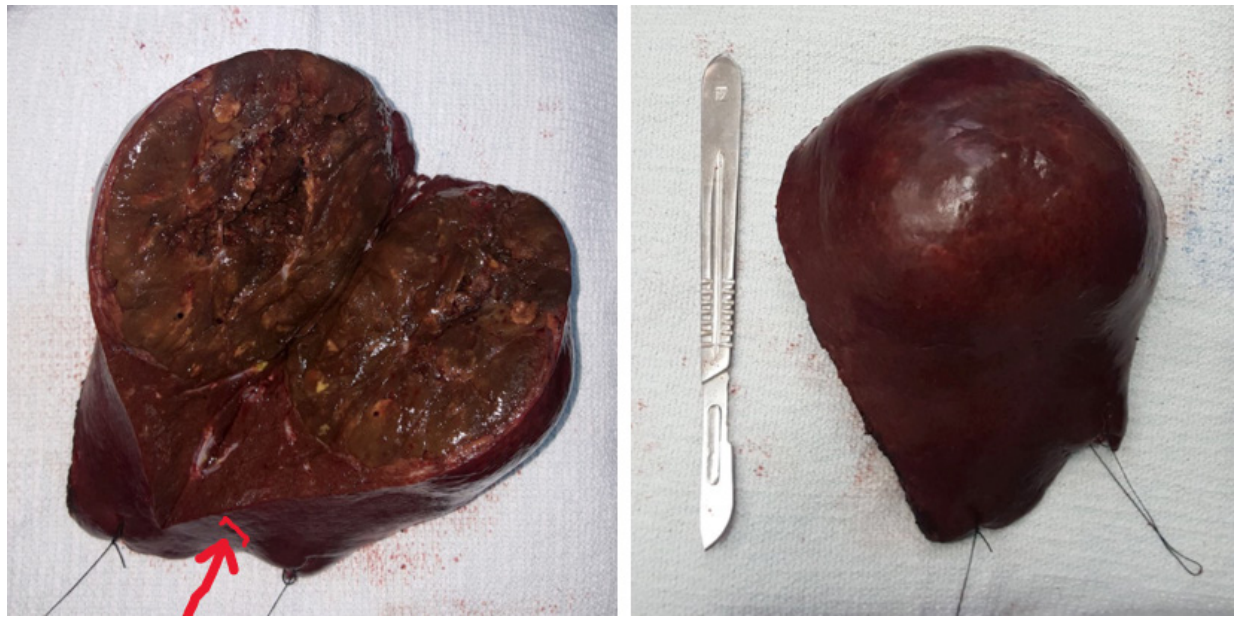

Figuras 3. Pieza quirúrgica producto de mesohepatectomía, margen microscópico de $1 \mathrm{~cm}$.

Después de la cirugía ingresó a la unidad de cuidados intensivos (UCI), extubado, sin soporte vasopresor. Presentó un pico isquémico moderado en el primer día postoperatorio sin alteración de la capacidad funcional del hígado. Su estancia hospitalaria total fue de cuatro días, dos de ellos en la UCI. No presentó ninguna complicación, ni reingreso temprano.

\section{Caso 2}

Masculino de 77 años, con antecedente de HTA y diabetes mellitus tipo 2 (DM2) insulinorrequiriente, quien consultó por dolor abdominal asociado a pérdida de cuatro kilos de peso. Se realizó TC de abdomen contrastado qué informó masa hepática de $8 \mathrm{~cm}$ en los segmentos 4,5 y 8 , y la biopsia con- firmó hepatocarcinoma estadio III de Edmondson. Se realizó RNM de abdomen (figura 4) que reportó compresión extrínseca de la confluencia de los hepáticos, obstrucción y dilatación del ramo biliar hacia el segmento 5, acorde con los hallazgos tomográficos. Se completó la estadificación mediante TC de cráneo y tórax, y gammagrafía ósea que fueron negativos para extensión metastásica. Entre los exámenes se destacan, AST de $64 \mathrm{U} / \mathrm{L}$, ALT de $54 \mathrm{U} / \mathrm{L}$, BT 0,55 mg/dL, FA de $131 \mathrm{U} / \mathrm{L}$, AFP $>3000 \mathrm{ng} / \mathrm{mL}$. Por el tamaño y la AFP elevada, se consideró que una embolización portal preoperatoria y la espera de seis semanas para generar hipertrofia compensadora representaba un alto riesgo de progresión, por lo que se decidió ofrecer una mesohepatectomía.

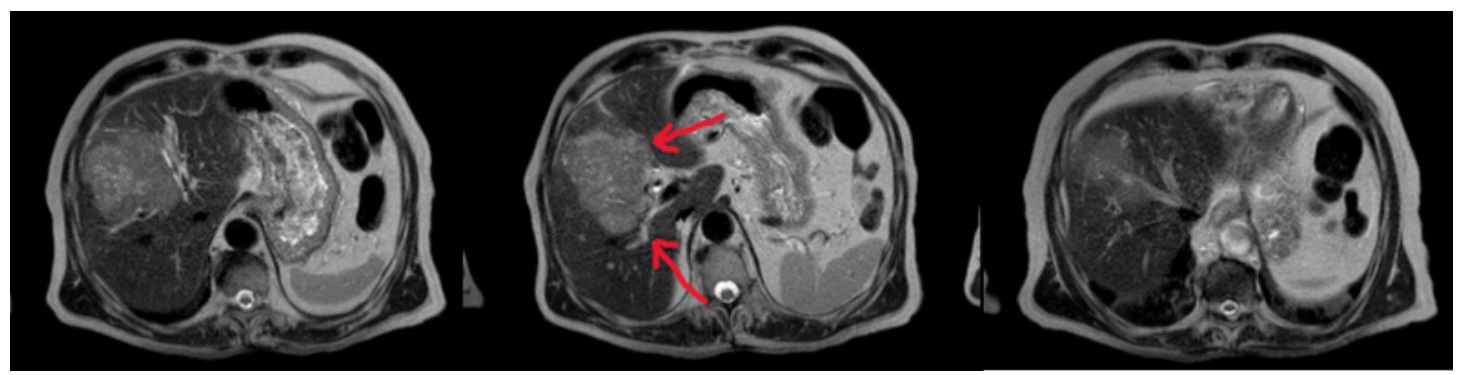

Figura 4. Masa hepática de $8 \mathrm{~cm}$ en los segmentos 4,5 y 8 . Se muestra el compromiso de la vía biliar hacia el sector lateral izquierdo y del sector posterior derecho (señalado en rojo). 
Tras una incisión y abordaje similar al paciente del caso número 1 , se disecaron y ligaron las ramas portales izquierdas hacia el segmento 4 por fuera de la cápsula de Glisson a través del espacio de Rex, causando la demarcación isquémica, y también se ligó la rama arterial proveniente de la arteria hepática izquierda (figuras 5 y 6). Mediante el uso del Cavitron Ultrasonic Surgical Aspirator (CUSA) y del Harmonic ${ }^{\circledR}$, se procedió con la resección hepática sobre el borde lateral del sector anterior y del borde medial del sector medial, preservando el drenaje venoso de los re-

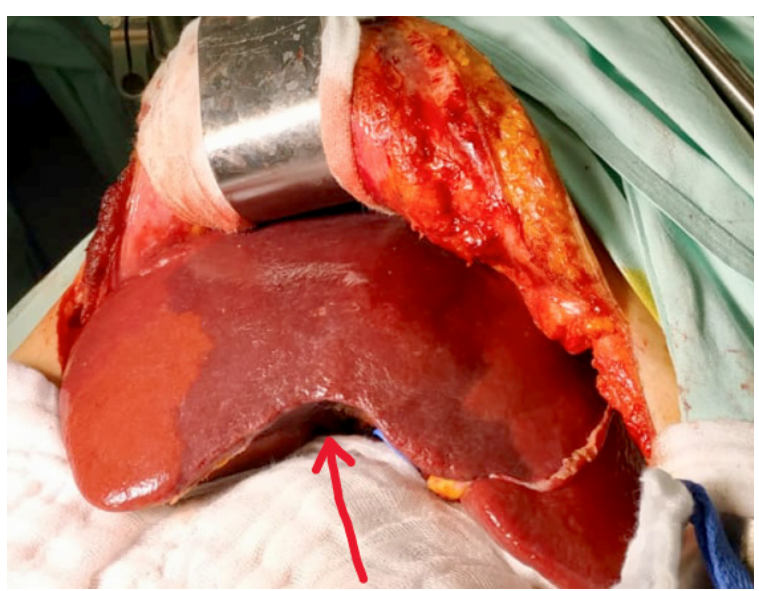

Figura 5. Demarcación isquémica del sector anterior derecho (flecha roja).

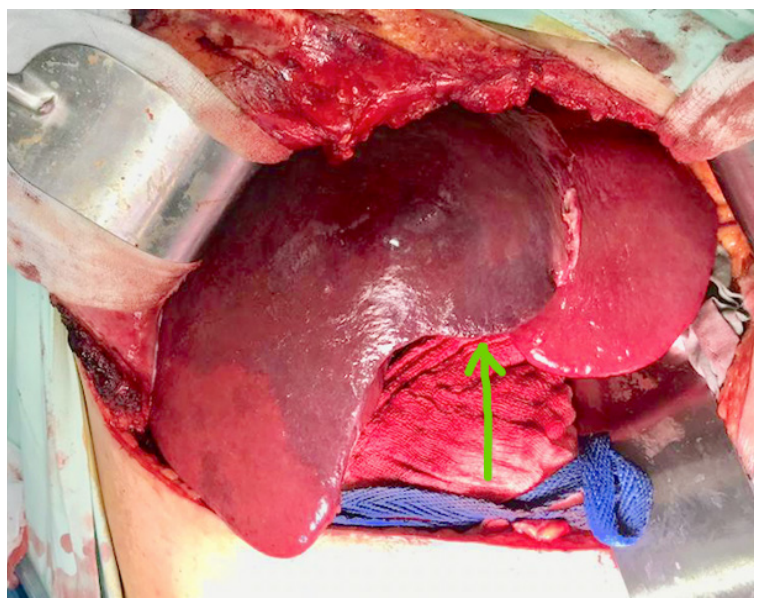

Figura 6. Demarcación isquémica de los sectores centrales: medial izquierdo (flecha verde) y anterior derecho. manentes hepáticos (venas suprahepáticas desde el sector lateral izquierdo y del sector posterior derecho); la ligadura de la vena hepática media se realizó con sutura mecánica.

Debido al compromiso neoplásico de la vía biliar, se resecó y se reconstruyó mediante una colangioyeyunostomía de ductos separados en $\mathrm{Y}$ de Roux (figura 7). El tiempo quirúrgico fue de 480 minutos, el sangrado de $1000 \mathrm{ml}$, y el tiempo de la maniobra de Pringle de 43 minutos (cuatro pinzamientos). El paciente presentó fuga biliar tipo B que se manejó con drenaje percutáneo y antibióticos de amplio espectro por 14 días. La hospitalización duro 23 días, 12 de ellos en cuidado crítico.

\section{Caso 3}

Paciente masculino de 66 años, con dolor abdominal e ictericia de dos semanas, asociado a fiebre y vómito. La ecografía mostró un hígado de contorno regular, sin evidencia de hepatopatía crónica, con una lesión compatible con hepatocarcinoma de 10,3 x 7 × 9,5 cm, comprometiendo los segmentos $4 \mathrm{a}, 5$ y 8, sin lesiones metastásicas (figura 8), y la biopsia percutánea de hígado corroboró el diagnóstico de hepatocarcinoma. Las pruebas de función hepática informaron: AST 42 U/L, ALT 46

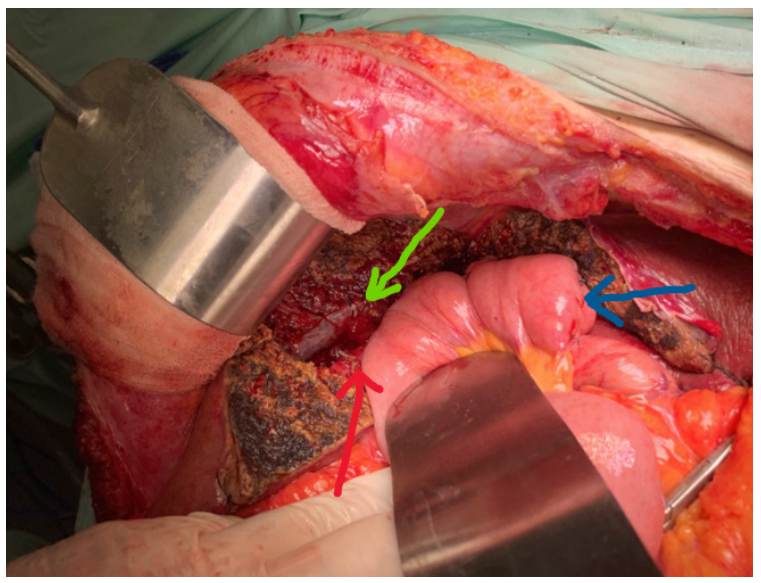

Figura 7. Reconstrucción en $Y$ de Roux por medio de colangio-yeyunostomía de ductos sector posterior derecho (flecha verde) y lateral izquierdo (flecha azul) por separado. Vena Suprahepática derecha completamente disecada luego de la resección del sector anterior derecho. 
U/L, BT 0,48 mg/dL, gamma glutamiltranspeptidasa (GGT) $85 \mathrm{U} / \mathrm{L}$, albúmina 3,9 g/dL, plaquetas 494,000 mcL. AntiHBs, HBsAg, HBc y antiHCV negativos; CA 19-9: < 2 u/mL, ACE $1 \mathrm{ng} / \mathrm{mL}$, AFP $11 \mathrm{ng} / \mathrm{mL}$. La TC de tórax descartó compromiso extra-abdominal.

Se llevó en primera instancia a embolización supraselectiva portal de ramas de los segmentos comprometidos (figura 9). La nueva estadificación dos semanas después no mostró progresión tumoral, y tras una adecuada valoración del riesgo quirúrgico se llevó a cirugía.

Por medio de una incisión Mackuuchi, se identificó la lesión sin compromiso extrahepático (figura 10), se liberaron los ligamentos hepáticos, se realizó colecistectomía, disección y control de la arteria hepática propia y de las venas hepáticas izquierda y derecha, y disección y pinzamiento de las ramas arteriales y portales del sector anterior derecho. La resección hepática se realizó con Harmonic ${ }^{\circledR}$ medial a la vena porta derecha y con hemostasia mediante ligadura de las ramas segmentarias (figura 11). Se continuó el control vascular de la arteria y la porta izquierda y resección hepática medial a la vena hepática y porta izquierda con igual técnica. El tiempo quirúrgico fue de 270 minutos y el sangrado de $2000 \mathrm{ml}$. El paciente fue traslado a UCI con soporte vasoactivo y ventilatorio que fueron retirados 48 horas luego, y con evolución adecuada se dio egreso hospitalario ocho días después.

\section{Resultados}

Tres pacientes masculinos con edad promedio de 75 años, con carcinoma hepático de localización central (sectores medial izquierdo y anterior derecho) y sin cirrosis, fueron llevados a cirugía con intención curativa. La media del tiempo operatorio fue de 310 minutos, el tiempo promedio de la maniobra de Pringle de 29 minutos; y el sangrado promedio de $1266 \mathrm{ml}$. Solo un paciente requirió transfusión (Tablas 1 y 2).

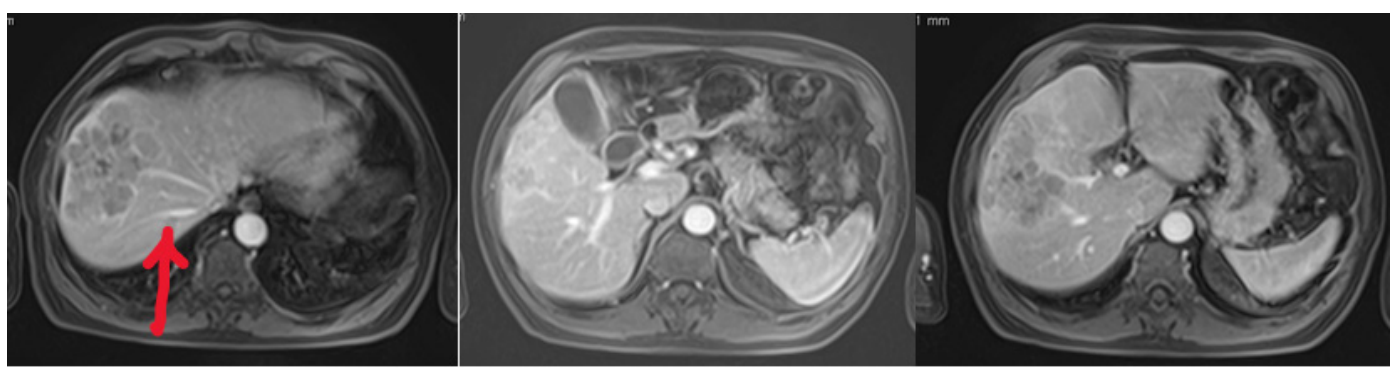

Figura 8. Hepatocarcinoma de 10,3 x 7 x 9,5 cm comprometiendo los segmentos 4a, 5 y 8, con preservación de vena suprahepática derecha en su recorrido (flecha roja).

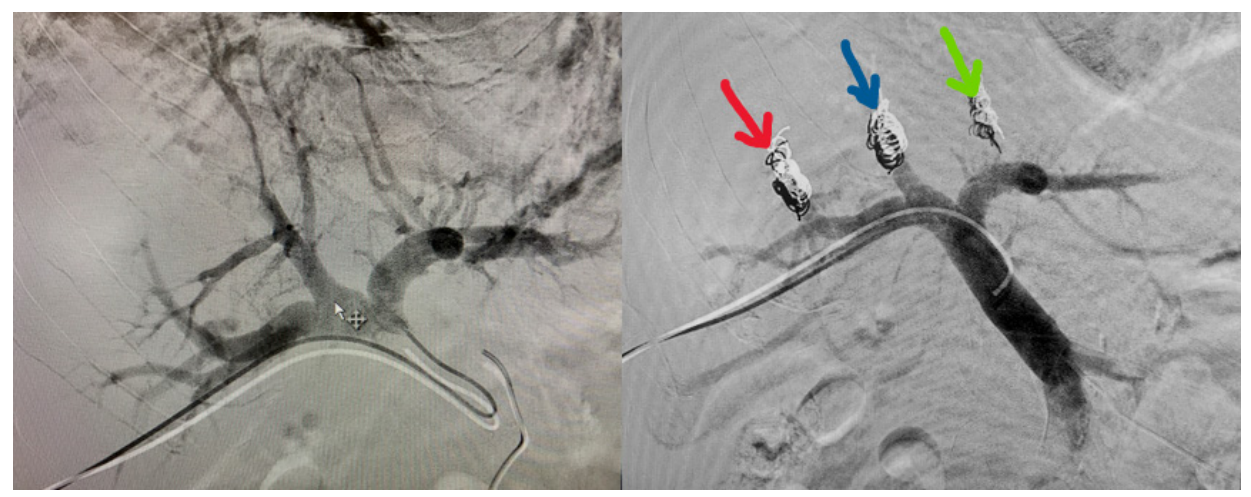

Figura 9. Embolización supra selectiva de ramos portales hacia sectores centrales. En rojo ramo portal accesorio hacia sector anterior derecho, en azul ramo venoso del sector anterior derecho, en verde ramos portales izquierdos hacia segmento 4 . 


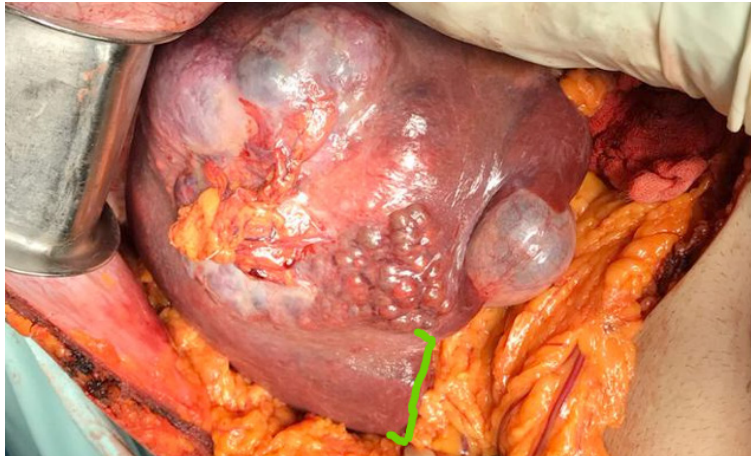

Figura 10. Hepatocarcinoma, hígado sin aspecto cirrótico ni compromiso regional. Sector posterior derecho libre de tumor (señalado en verde).

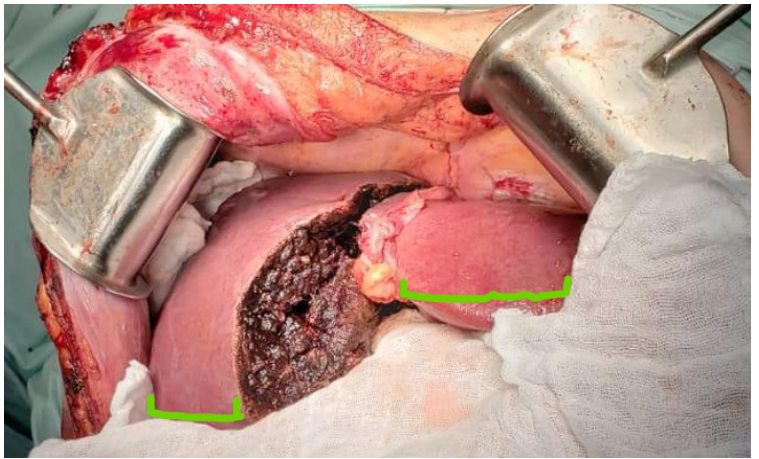

Figura 11. Resección hepática de sectores centrales medial izquierdo y anterior derecho. Preservación del sector posterior derecho y lateral izquierdo (señalado en verde).

Tabla 1 Características demográficas y quirúrgicas de los pacientes.

\begin{tabular}{lccccccc}
\hline Sexo & Edad & $\begin{array}{c}\text { Duración } \\
\text { Cirugía } \\
\text { (en minutos) }\end{array}$ & $\begin{array}{c}\text { Sangrado en } \\
\text { mililitros }\end{array}$ & $\begin{array}{c}\text { Duración } \\
\text { maniobra de } \\
\text { Pringle } \\
\text { (en minutos) }\end{array}$ & $\begin{array}{c}\text { Transfusión } \\
\text { glóbulos rojos } \\
\text { empaquetados }\end{array}$ & $\begin{array}{c}\text { Hospitalización } \\
\text { UCl/Total } \\
\text { (en días) }\end{array}$ & Complicaciones \\
\hline Masculino & 83 & 180 & 800 & 16 & No & $2 / 4$ & No \\
Masculino & 77 & 480 & 1000 & 43 & No & $12 / 23$ & $\begin{array}{c}\text { Si, fuga biliar } \\
\text { tipo B }\end{array}$ \\
Masculino & 66 & 270 & 2000 & No & 3 unidades & $2 / 8$ & No \\
\hline
\end{tabular}

Tabla 2 Características histopatológicas de los hepatocarcinomas resecados

\begin{tabular}{lccccc}
\hline Tipo Histológico & $\begin{array}{c}\text { Clasificación } \\
\text { Edmondson }\end{array}$ & pTNcM & $\begin{array}{c}\text { Compromiso } \\
\text { Linfovascularl } \\
\text { Perineural }\end{array}$ & $\begin{array}{c}\text { Compromiso } \\
\text { de los bordes }\end{array}$ & $\begin{array}{c}\text { Distancia } \\
\text { del margen }\end{array}$ \\
\hline Carcinoma Hepatocelular & 1 & pT3pNxcM0 & $+/-$ & Libre & $5 \mathrm{~mm}$ \\
$\begin{array}{l}\text { Carcinoma Hepatocelular } \\
\begin{array}{l}\text { Carcinoma Hepatocelular } \\
\text { de tipo trabecular y }\end{array}\end{array}$ & 3 & pT2pN0cM0 & $+/+$ & Libre & $1 \mathrm{~mm}$ \\
pseudoganglionar & 2 & pT3pNOcM0 & $+/-$ & Libre & $1 \mathrm{~mm}$ \\
\hline
\end{tabular}

\section{Discusión}

\section{Evaluación funcional del hígado remanente.}

La evaluación de la función hepática se puede realizar mediante pruebas estáticas (bioquímicas, volumetría hepática y puntajes de graduación clínica) o dinámicas (basado en la captación y o el metabolismo de compuestos) ${ }^{9}$. Las pruebas bioquímicas (bilirrubinas, albumina y tiempo de protrombina) las imágenes y los sistemas de graduación clínica (puntaje de Child-Pugh y MELD) son de gran utilidad en el preoperatorio para establecer si el hígado es sano o cirrótico, y en pacientes con hepatocarcinoma en hígado cirrótico permiten determinar el tratamiento entre el trasplante o la resección ${ }^{10}$. Sin embargo, se ha demostrado el bajo rendimiento predictor del Child-Pugh para muerte por falla hepática posterior a hepatectomía, y del MELD, con una sensibilidad para morbilidad del 51 $\%$ y mortalidad del $70 \%$ en pacientes con hígado no cirrótico ${ }^{11}$. 
Las imágenes como la tomografía o la resonancia nuclear magnética de abdomen son de mayor importancia porque ayudan a evaluar las características morfológicas del hígado, signos indirectos de hepatopatía crónica (nodularidad de los bordes, atrofia o hipertrofia de los lóbulos, esplenomegalia, circulación colateral, permeabilidad y tamaño de la porta) y determinar el volumen remanente posterior a la resección. Volúmenes residuales funcionales por encima del $25 \%$ en paciente con hígado sano tienen menor riesgo de complicaciones mayores, particularmente de falla hepática posterior a hepatectomía, en cambio en pacientes con hígado cirrótico o enfermo (hígado graso o posterior a quimioterapia) se deben dejar volúmenes residuales del $40 \%$ o más, para disminuir el riesgo de este tipo de complicaciones ${ }^{12}$, ${ }^{13}$. En nuestros pacientes utilizamos las medidas estáticas para evaluar la función hepática y no encontramos ningún signo de cirrosis ni de hepatopatía crónica.

Gracias a los desarrollos en trasplante hepático de donante vivo se han establecido volúmenes promedios de cada uno de los lóbulos o sectores del hígado, como el sector lateral izquierdo (segmento 2 y 3 ) o el sector posterior derecho (segmentos 6 y 7) que corresponden cada uno al $20 \%$ del volumen total del hígado, es decir a un volumen entre 200 y $300 \mathrm{ml}^{14,15}$. Por otro lado, al comparar el volumen de resección, la media del volumen fue menor para la mesohepatectomía (560 ml), comparado con la lobectomía (900 ml) y con la hepatectomía ampliada $(1500 \mathrm{ml}){ }^{16}$. En este estudio no se realizó volumetría específica de los sectores remanentes, teniendo en cuenta que los pacientes no presentaban cirrosis y bajo el entendido de que las resecciones realizadas dejarían un porcentaje de volumen superior al de una hepatectomía extendida que corresponde al $20 \%$ del volumen total del hígado.

El empleo de herramientas dinámicas como la depuración del verde de indocianina y la capacidad de eliminación de la galactosa ayudan a establecer la funcionalidad en tiempo real de la totalidad del hígado ${ }^{9}$, sin embargo ninguna de esas pruebas ha mostrado con gran precisión la función del remanente ${ }^{17}$. En nuestra serie no se realizaron pruebas dinámicas debido a que no están disponibles en nuestro medio.

\section{Mesohepatectomía versus hepatectomía extendida}

La mesohepatectomía es una herramienta terapéutica muy útil que, evita resecciones hepáticas extendidas en pacientes con tumores centrales, conservando así un mayor volumen hepático residual de entre el 35 y el $50 \%$ y disminuyendo el riesgo de falla hepática posterior a hepatectomía ${ }^{18}$. Series comparativas entre MH y HE señalan una morbilidad del 5,6\% para MH comparada con el $14 \%$ en hepatectomía ampliada ${ }^{16}$.

En pacientes con excelente función hepática, un volumen residual hepático (VRH) mayor del $25 \%$ es adecuado para prevenir la falla hepática. Esto fue validado en el estudio realizado por Kishi, quien demostró que un VRH menor o igual al $20 \%$ es un factor independiente para falla hepática posterior a hepatectomía (FHPH), con un RR de 1,72 - 7,99 ${ }^{19}$. En la literatura se describe una tasa de FHPH de 2,9\% para la mesohepatectomía, y del 7,3 \% para hepatectomías extendidas ${ }^{20}$. Ninguno de nuestros pacientes presentó FHPH.

Hasta el momento no se dispone de estudios aleatorizados que demuestren la eficiencia de la mesohepatectomía, pero existen algunas comparaciones de estudios no aleatorizados, en donde se puede observar una mortalidad que va de 0 al 6,25\%, siendo la causa más frecuente la falla hepática y el sangrado ${ }^{2}$. En nuestros pacientes no ocurrió mortalidad peri-operatoria.

En la revisión sistémica de Li y colaboradores se analizaron desenlaces fuertes comparando MH y HE. La pérdida de sangre fue mayor para la $\mathrm{MH}$ con $1156 \mathrm{ml}$ versus $593 \mathrm{ml}$ en la $\mathrm{HE}^{20}$, lo que se correlaciona bien con nuestros casos pues el sangrado promedio fue de $1000 \mathrm{ml}$. En cuanto al tiempo operatorio, este es mayor para las $\mathrm{MH}$ comparado con la HE, debido a la mayor dificultad técnica de disección vascular y por incluir dos secciones hepáticas. En el estudio mencionado se reportó una media de tiempo operatorio de 311 versus 276 minutos (MH vs HE), con una diferencia estadísticamente significativa $(\mathrm{p}<0,001)$, siendo igual a lo reportado en nuestros pacientes. 
De igual manera, el tiempo de oclusión vascular (maniobra de Pringle) fue mayor para la $\mathrm{MH}(38,2$ min) que para la HE $(32,4 \mathrm{~min})^{21}$, y notoriamente menor que el tiempo de maniobra en nuestros pacientes.

En las cirugías de resección hepática, las fugas biliares se reportaron entre $3 \%-17 \%{ }^{22}$, y para su clasificación se tienen en cuenta los criterios del International Study Group of Liver Surgery (ISGLS) ${ }^{23}$. En nuestro caso, solo un paciente tuvo una fuga biliar clasificada como tipo B. En la revisión sistemática de Li se encontró una tasa de fuga biliar del 13,5\% para las $\mathrm{MH}$ y del 6,7 \% para las HE. En ambas técnicas la ascitis es informada hasta en un $18 \%$ de los ca$\operatorname{sos}^{20}$, mientras en nuestra serie ningún paciente presentó ascitis. En la mesohepatectomía otras causas de morbilidad peri-operatoria como la infección de la herida quirúrgica $(1,7$ - 6,2\%), el absceso intrabdominal $(0,8-1,9 \%)$, la neumonía asociada al cuidado de la salud $(1,7-12,5 \%)$ y los empiemas ( 5,7 a $23,5 \%)$, pueden hacer que la morbilidad de este tipo de procedimientos ascienda hasta el $55 \%{ }^{2}$.

La selección de pacientes es un factor fundamental para los buenos resultados, ya que de ello depende en gran medida la mayor o menor probabilidad de complicaciones. Si bien los pacientes sin cirrosis son candidatos ideales, la mesohepatectomía puede ser realizada de forma segura en paciente con cirrosis. En la publicación de Chen y colaboradores, el $92 \%$ de sus pacientes tenían cirrosis Child-Pugh A, con un remanente hepático suficiente, mayor del $45 \%$ del volumen antes de resección ${ }^{24}$. Sin embargo, ante un paciente con hepatocarcinoma en hígado cirrótico, la recomendación es ofrecerle un trasplante hepático si cumple con la edad y los criterios de Milán ${ }^{10}$. En este trabajo ninguno de los pacientes cumplía criterios de Milán y todos eran mayores de 65 años, por lo que no fueron considerados para trasplante. Por último, el hecho de dejar mayor remanente hepático durante la mesohepatectomía, permite realizar a largo plazo, nueva resección de recurrencias en hígado residual, el cual es el tratamiento de elección de dichos tumores en hígado no cirrótico ${ }^{10,25}$.

\section{Conclusiones}

La mesohepatectomía es una alternativa técnicamente más demandante que una hepatectomía extendida, tiene mayor riesgo de sangrado intraoperatorio y de fugas biliares del lecho de transección, por lo que muchos centros la han dejado como una alternativa casi anecdótica para el tratamiento de tumores de segmentos centrales, sin embargo, es una técnica reproducible y tiene mucho menor riesgo de desencadenar una falla hepática posterior a hepatectomía.

\section{Cumplimiento de normas éticas}

Consentimiento informado: El diseño y la metodología de este estudio fueron aprobados por el Comité de Ética Institucional, y se contó con el consentimiento informando firmado por parte de los pacientes.

Conflictos de interés: Ninguno declarado por los autores.

Fuente de financiación: Financiado con recursos propios de los autores.

\section{Contribución de los autores:}

Concepción y diseño del estudio: Luis Manuel BarreraLozano, Cristian Leonardo Muñoz.

Adquisición de datos: Luis Manuel Barrera-Lozano, Diana Catalina Pineda.

Análisis e interpretación de datos: Luis Manuel BarreraLozano, Cristian Leonardo Muñoz, Diana Catalina Pineda, Jorge Andrés Becerra, Jaime Alberto Ramírez.

Redacción del manuscrito: Luis Manuel Barrera-Lozano, Cristian Leonardo Muñoz.

Revisión crítica: Luis Manuel Barrera-Lozano, Diana Catalina Pineda, Jorge Andrés Becerra, Jaime Alberto Ramírez, Jorge lván Gutiérrez-Montoya.

\section{Referencias}

1. McBride CM, Wallace S. Cancer of the right lobe of the liver: a variety of operative procedures. Arch Surg. 1972;105:289-96.

https://doi.org/10.1001/archsurg.1972.04180080139023

2. Stratopoulos C, Soonawalla Z, Brockmann J, Hoffmann K, Friend PJ. Central hepatectomy: the golden mean for treating central liver tumors? Surg Oncol. 2007;16:99106. https://doi.org/10.1016/j.suronc.2007.05.002

3. Strasberg SM. Nomenclature of hepatic anatomy and resections: a review of the Brisbane 2000 system. J 
Hepatobiliary Pancreat Surg. 2005;12:351-5. https://doi.org/10.1007/s00534-005-0999-7

4. Lowe MC, D’Angelica MI. Anatomy of hepatic resectional surgery. Surg Clin North Am. 2016;96:183-95. https://doi.org/10.1016/j.suc.2015.11.003

5. Wu CC, Ho WL, Chen JT, Tang CS, Yeh DC, Liu TJ, et al. Mesohepatectomy for centrally located hepatocellular carcinoma: an appraisal of a rare procedure. J Am Coll Surg. 1999;188:508-15.

https://doi.org/10.1016/S1072-7515(99)00026-5

6. Herman P, Machado MAC. Meso-hepatectomia: uma alternativa para a ressecção hepática alargada. Rev Colégio Bras Cir. 2005;32:90-3.

https://doi.org/10.1590/S0100-69912005000200009

7. Araujo RLC, Cesconetto D, Jeismann VB, Fonseca GM, Coelho FF, Kruger JAP, et al. Hepatectomia central para cistoadenoma biliar: preservação de parênquima em lesões benignas. ABCD Arq Bras Cir Dig São Paulo. 2016;29:295-6.

https://doi.org/10.35509/01239015.468

8. Sanz LEM, Endis M, Llanos F, Chimbo J, Abril X, Faican F, et al. Hepatectomía central combinada con ligadura de la vena porta derecha en un niño con hepatoblastoma bilobar: reporte de un caso. Rev Colomb Cancerol. 2020;24:195-9.

https://doi.org/10.35509/01239015.468

9. Hoekstra LT, de Graaf W, Nibourg GAA, Heger M, Bennink RJ, Stieger B, et al. Physiological and biochemical basis of clinical liver function tests: a review. Ann Surg. 2013;257:27-36.

https://doi.org/10.1097/SLA.0b013e31825d5d47

10. Hoekstra L. De Graaf W. Nibourg G. Heger M. Bennink R. Stieger B, et al. Physiological and biochemical basis of clinical liver function tests. Ann Surg. 2013;257:27-36. https://doi.org/10.1097/SLA.0b013e31825d5d47

11. Ray S, Mehta NN, Golhar A, Nundy S. Post hepatectomy liver failure - A comprehensive review of current concepts and controversies. Ann Med Surg. 2018;34:4-10. https://doi.org/10.1016/j.amsu.2018.08.012

12. Shoup M, Gonen M, D'Angelica M, Jarnagin WR, DeMatteo RP, Schwartz LH, et al. Volumetric analysis predicts hepatic dysfunction in patients undergoing major liver resection. J Gastrointest Surg Off J Soc Surg Aliment Tract. 2003;7:325-30. https://doi.org/10.1016/S1091-255X(02)00370-0

13. Vauthey JN, Chaoui A, Do KA, Bilimoria MM, Fenstermacher MJ, Charnsangavej C, et al. Standardized measurement of the future liver remnant prior to extended liver resection: methodology and clinical associations. Surgery. 2000;127:512-9. https://doi.org/10.1067/msy.2000.105294

14. Weyker PD, Brentjens TE. Anesthetic aspects of living donor hepatectomy. En: Wagener G, editor. Liver Anesthesiology and Critical Care Medicine. Springer Inter- national Publishing; 2018 p. 367-75. Disponible en: https://doi.org/10.1007/978-3-319-64298-7_28

15. Trotter JF, Kam I. Living donor transplantation. En: Elsevier. Transplantation of the Liver. Disponible en: https://doi.org/10.1016/B978-1-4557-0268-8.00049-X

16. Scudamore CH, Buczkowski AK, Shayan H, Ho SG, Legiehn GM, Chung SW, et al. Mesohepatectomy. Am J Surg. 2000;179:356-60. https://doi.org/10.1016/S0002-9610(00)00374-3

17. Fan ST. Liver functional reserve estimation: state of the art and relevance for local treatments: the Eastern perspective. J Hepato-Biliary-Pancreat Sci. 2010;17:380-4. https://doi.org/10.1007/s00534-009-0229-9

18. Mehrabi A, Mood ZA, Mood Z, Roshanaei N, Fonouni $\mathrm{H}$, Müller SA, et al. Mesohepatectomy as an option for the treatment of central liver tumors. J Am Coll Surg. 2008;207:499-509. https://doi.org/10.1016/j.jamcollsurg.2008.05.024

19. Kishi Y, Abdalla EK, Chun YS, Zorzi D, Madoff DC, Wallace MJ, et al. Three hundred and one consecutive extended right hepatectomies: evaluation of outcome based on systematic liver volumetry. Ann Surg. 2009;250:540-8. https://doi.org/10.1097/SLA.0b013e3181b674df

20. Li J, Wang C, Song J, Chen N, Jiang L, Yang J, et al. Mesohepatectomy versus extended hemihepatectomies for centrally located liver tumors: a meta-analysis. Sci Rep. 2017;7:9329. https://doi.org/10.1038/s41598-017-09535-0

21. Li W, Li L, Minigalin D, Wu H. Anatomic mesohepatectomy versus extended hepatectomy for patients with centrally located hepatocellular carcinoma. HPB. 2018;20:530-7.

https://doi.org/10.1016/j.hpb.2017.11.012

22. Ishii M, Mizuguchi T, Harada K, Ota S, Meguro M, Ueki T, et al. Comprehensive review of post-liver resection surgical complications and a new universal classification and grading system. World J Hepatol. 2014;6:745-51. https://doi.org/10.4254/wjh.v6.i10.745

23. Koch M, Garden OJ, Padbury R, Rahbari NN, Adam R, Capussotti L, et al. Bile leakage after hepatobiliary and pancreatic surgery: a definition and grading of severity by the International Study Group of Liver Surgery. Surgery. 2011;149:680-8.

https://doi.org/10.1016/j.surg.2010.12.002

24. Chen X-P, Qiu F-Z, Lau W-Y, Zhang B-X, Chen Y-F, Zhang W-G, et al. Mesohepatectomy for hepatocellular carcinoma: a study of 256 patients. Int J Colorectal Dis. 2008;23:543-6. https://doi.org/10.1007/s00384-007-0411-y

25. Mergental H, Adam R, Ericzon B-G, Kalicinski P, Mühlbacher F, Höckerstedt $\mathrm{K}$, et al. Liver transplantation for unresectable hepatocellular carcinoma in normal livers. J Hepatol. 2012;57:297-305. https://doi.org/10.1016/j.jhep.2012.03.022 